\title{
Evaluation of local immune response to Fasciola hepatica experimental infection in the liver and hepatic lymph nodes of goats immunized with Sm14 vaccine antigen
}

\author{
Ricardo E Mendes' ${ }^{1}$ Rafael Zafra', Rafael A Pérez-Écija1, Leandro Buffoni², \\ Álvaro Martínez-Moreno², Miriam Tendler³, José Pérez¹/+ \\ 1Departamento de Anatomía y Anatomía Patológica Comparadas ²Departamento de Sanidad Animal (Parasitología), Facultad de \\ Veterinaria, Universidad de Córdoba, Carretera Madrid-Cádiz Km 396, 14014 Córdoba, Spain ${ }^{3}$ Laboratório de Esquistossomose \\ Experimental, Fundação Oswaldo Cruz-Fiocruz, Rio de Janeiro, RJ, Brasil
}

Protection against Fasciola hepatica in goats immunized with a synthetic recombinant antigen from Schistosoma mansoni fatty acid-binding protein 14 (rSm14) was investigated by assessing worm burdens, serum levels of hepatic enzymes, faecal egg count and hepatic damage, which was evaluated using gross and microscopic morphometric observation. The nature of the local immune response was assessed by examining the distribution of $\mathrm{CD}^{+}, \mathrm{CD} 4^{+}$, $C D 8^{+}$and $\gamma \delta^{+}$T lymphocytes along with $I g G^{+}, I L-4^{+}$and $I F N-\gamma^{+}$cells in the liver and hepatic lymph nodes (HLN). The goats used consisted of group 1 (unimmunized and uninfected), group 2 [infected control - immunized with Quillaia A (Quil A)] and group 3 (immunized with $r S m 14$ in Quil A and infected), each containing seven animals. Immunization with rSm14 in Quil A adjuvant induced a reduction in gross hepatic lesions of $56.6 \%(p<0.001)$ and reduced

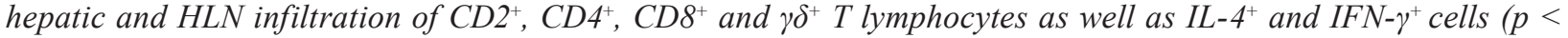
$0.05)$. This is the first report of caprine immunization against $\mathrm{F}$. hepatica using a complete $r S m 14$ molecule derived from S. mansoni. Immunization reduced hepatic damage and local inflammatory infiltration into the liver and HLN. However, considering that Quil A is not the preferential/first choice adjuvant for Sm14 immunization, further studies will be undertaken using the monophosphoryl lipid A-based family of adjuvants during clinical trials to facilitate anti-Fasciola vaccine development.

Key words: Sm14 - Fasciola hepatica - vaccines - goat - liver - parasite infection

Fasciolosis, caused by Fasciola hepatica, is the most important parasitic livestock disease and an economically important disease in ruminants in Europe, the Americas, Australia and New Zealand. Fasciolosis represents a recognized, unsolved agricultural problem responsible for economic losses estimated at around US\$ 3 billion per year (Spithill et al. 1999). Recent reports indicate that it is also a major human pathogen and is increasingly being recognized as a cause of significant human diseases (Mas-Coma et al. 2005).

Liver fluke infections can be highly pathogenic and can lead to severe morbidity and even death of the host. The flukicide triclabendazole is still the drug of choice used in Fasciola control programs (Overend \& Bowen 1995); however, the high cost of treatment prevents widespread use by rural producers in developing countries. Moreover, resistance to triclabendazole has been reported in sheep infected with $F$. hepatica (Overend \& Bowen 1995, Moll et al. 2000), suggesting that selection of resistant parasites may eventually compromise the use of this drug. Furthermore, although chemotherapy

\footnotetext{
Financial support: EUproject (FOOD-CT-2005-023025-DELIVER) + Corresponding author: an1pearj@uco.es

Received 14 January 2010

Accepted 29 April 2010
}

has been used for more than two decades with some efficacy in reducing morbidity rates, it represents only a palliative measure, leaving transmission rates unaltered due to continuous re-infection in endemic areas (Spithill et al. 1999, Dalton et al. 2003, Meeusen \& Piedrafita 2003). Vaccines represent the most attractive long-term alternative to solve this dilemma, as they represent an environmentally-friendly method for the control of liver fluke disease in livestock.

Indeed, the prevalence of fasciolosis in livestock has increased dramatically in the last few years. For example, up to 12 -fold increases in infection rates have been observed in several European Union member states. In the United Kingdom, the prevalence of infection in cattle ranges from $45-84 \%$ and in Ireland alone, annual losses have been estimated at $€ 60$ million (Tendler \& Simpson 2008).

Focusing on molecular characterization of secretorexcretory (SE) components with the goal of identifying immunoprotective components contained therein, the recombinant antigen from Schistosoma mansoni fatty acid-binding protein 14 (rSm14) molecule was selected from a mixture of adult schistosome components obtained from living worms that have been previously shown to protect mice against infection. This molecule was identified by a long-term investigation focusing on vaccination experiments in populations of out-bred animals and further characterized by gene cloning, producing a $14 \mathrm{kDa}$ molecule named Sm14, belonging to a 
family of fatty-acid binding proteins (Moser et al. 1991, Tendler et al. 1996). It has been previously demonstrated that Sm14 can induce high levels of SE antigens against $S$. mansoni in experimental infections and is also protective against $F$. hepatica (Tendler et al. 1995, 1996).

$\mathrm{rSm} 14$ is now being actively developed as a bivalent anti-helminth vaccine for use in both livestock disease caused by $F$. hepatica and human endemic schistosomiasis (Tendler \& Simpson 2008).

The aim of this study was to evaluate protection against $F$. hepatica in goats immunized with $\mathrm{rSm} 14$ by assessing worm burdens, serum levels of hepatic enzymes, faecal eggs counts, gross and microscopic hepatic lesions and the local immune response.

\section{MATERIALS AND METHODS}

Experimental design - This study consisted of a 10 week period of immunization followed by an experimental infection (17 weeks) and subsequent sacrifice of all animals, 27 weeks in all. Animals were first acclimatized for 30 days to the experimental conditions.

The sample included 21 Florida Sevillana goats. Animals were four months of age and free of parasitic and infectious diseases, as indicated by faecal analysis and absence of clinical signs. Animals were randomly allocated into three groups of seven each. Group 1 was used as an uninfected control. The other two groups were immunized twice, on the 1st and 4th week. The 2 nd group was used as an infection control and injected with $1 \mathrm{~mL}$ of phosphate buffered saline (PBS) in $1 \mathrm{~mL}$ of adjuvant Quillaia A (Quil A). The 3rd group was immunized with $100 \mu \mathrm{g}$ of rSm14 in $1 \mathrm{~mL}$ of Quil A with quantum satis of $2 \mathrm{~mL}$ of PBS. rSm14 was kindly provided by Prof. Miriam Tendler from the Oswaldo Cruz Foundation, Brazil.

In the 10th week, groups 2 and 3 were infected with 200 F. hepatica metacercariae of ovine origin (Bristol University, Bristol, UK). The doses were administered orally in gelatine capsules. Infected and control goats were killed by intravenous injection of thiobarbital 17 weeks after infection and subjected to necropsy. The experiment was approved by the Bioethical Committee of the University of Cordoba (7119) and was carried out taking into account European (86/609/CEE) and Spanish (RD 223/1988) directives for animal experimentation. Two animals from group 3 died on day 4 after infection due to acute rumenitis that was unrelated to the infection.

Faecal egg count and fluke burdens - Faecal egg counts were carried out using the sedimentation technique as described previously (Mendes et al. 2010).

Plasma levels of hepatic enzymes - Blood was collected during the experiment to obtain serum for determination of liver enzymes gamma glutamyltransferase (GGT) and glutamate dehydrogenase (GLDH) as described previously (Mendes et al. 2010).

Histopathology - During necropsy, tissue samples were randomly collected from the left and right hepatic lobes, gallbladder and hepatic lymph nodes (HLN), fixed in $10 \%$ buffered formalin and embedded in paraffin wax. Tissue sections $(4 \mu \mathrm{m})$ were stained with haematoxylin and eosin for histopathological study. Four liver and two HLN tissue sections per animal were independently evaluated by two pathologists in a blind way and lesions were scored as follows: 0 : absent; 0.5 : mild; 1: moderate; 2 : severe and 3: very severe.

Morphometric studies - Morphometric studies were carried out using the Image-Pro Plus 4.0 software (Media Cybernetics, Silver Spring, MD, USA). Gross morphology was assessed to evaluate the percentage of damaged hepatic surface, as described previously (Zafra et al. 2008).

Microscopic liver morphology was examined to evaluate: (i) the area of portal spaces and microscopic hepatic damage (chronic tracts, cholangitis with eosinophils, lymphocytes and plasma cell infiltration, fibrosis and granulomas) and (ii) the area of bile ducts. Ten low-power microphotographs per animal were taken, each measuring $8.5 \mathrm{~mm}^{2}$. These microphotographs were randomly taken of damaged hepatic areas. First, hepatic damaged areas and portal spaces were delineated and the area was obtained. Second, the outer perimeter of bile ducts was delineated and the area was obtained. The results are expressed as percentage [mean \pm standard deviation (SD)] per group.

Microscopic morphology of HLN was assessed using six microphotographs of $8.5 \mathrm{~mm}^{2}$ each. In these photographs, medullar and capsular regions were delineated to obtain the cortical area. Then lymphoid follicles were delineated and the follicular and interfollicular areas were obtained. The results are expressed as the percentage of cortex occupied by lymphoid follicles (mean $\pm \mathrm{SD}$ ) group.

Immunohistochemistry - Tissue samples from the left and right hepatic lobes and HLN were snap-frozen in optimal cutting temperature (Miles, Elkhart, IN, USA) and immersed in liquid nitrogen-cooled 2-methylbutane (Merck, Darmstadt, Germany). Frozen samples were stored at $-80^{\circ} \mathrm{C}$ until they were cut in serial tissue sections of $5 \mu \mathrm{m}$ thickness using a cryostat at $-25^{\circ} \mathrm{C}$ and stored again at $-80^{\circ} \mathrm{C}$ until used.

The avidin-biotin-peroxidase (ABC) method was used for immunohistochemistry on snap-frozen tissue samples (Pérez et al. 1998). Endogenous peroxidase activity was blocked by incubation with $0.05 \%$ phenyl-hydrazine $\left(\right.$ Sigma $^{\circledR}$, St Louis, MO, USA) in PBS at $\mathrm{pH} 7.6$ for $30 \mathrm{~min}$. Tissue sections were incubated with $5 \%$ normal goat serum (Vector Laboratories, Burlingame, CA, USA) for $30 \mathrm{~min}$ at room temperature. Then primary antibodies were applied overnight $(18 \mathrm{~h})$ at $4^{\circ} \mathrm{C}$.

Details of primary antibodies are shown in Table I. Monoclonal antibodies (mAb) used for detecting lymphocyte subsets (mouse anti-bovine $\mathrm{CD} 2^{+}, \mathrm{CD}^{+}, \mathrm{CD}^{+}$, $\mathrm{TCR}^{+}$and $\mathrm{IgG}^{+}$) have shown cross-reactivity with caprine antigens (Navarro et al. 1996, Pérez et al. 1998). Mouse anti-bovine IL- $4^{+}$and IFN- $\gamma^{+}$mAbs cross react with caprine antigens according to the supplier's information. After three 10 min rinses in PBS, biotinylated goat antimouse immunoglobulin serum (Dako, Denmark) diluted 1:50 was applied to slides incubated with primary mAbs for $30 \mathrm{~min}$ and a goat anti-rabbit immunoglobulin serum (Dako, Denmark) diluted 1:200 was applied to slides incubated with the primary rabbit anti-goat $\operatorname{IgG}$ polyclonal antibody for $30 \mathrm{~min}$. 
TABLE I

Details of primary antibodies used, dilution and source

\begin{tabular}{lcccc}
\hline Antibody & Specificity & Origin & Dilution & Source \\
\hline BAQ95A & CD2 & Mouse & $1: 1000$ & VMRD Inc \\
GC50A1 & CD4 & Mouse & $1: 50$ & VMRD Inc \\
CACT80C & CD8 & Mouse & $1: 500$ & VMRD Inc \\
CACTB6A & $\gamma / \delta$ & Mouse & $1: 200$ & VMRD Inc \\
AI-5000 & IgG & Rabbit & $1: 1000$ & Vector \\
MCA1820 & IL-4 & Mouse & $1: 50$ & Serotec \\
MCA1783 & IFN- $\gamma$ & Mouse & $1: 50$ & Serotec
\end{tabular}

VMRD Inc, Pullman, WA, USA; Serotec, Oxford, UK; Vector Laboratories, Inc, Burlingame, CA, USA.

After two 10 min rinses in PBS, an $\mathrm{ABC}$ complex (Vector, Burlinghame, USA) diluted 1:50 was applied for $1 \mathrm{~h}$ as a $3 \mathrm{rd}$ reagent. Then tissue sections were incubated with 3,3'-diaminobenzidine tetrahydrochloride (Sigma) diluted to $0.035 \%$ in Tris-buffered saline ( $\mathrm{pH} 7.2$ ) with $0.1 \%$ hydrogen peroxide for $1 \mathrm{~min}$, rinsed in distilled water, lightly counterstained with Mayer's haematoxylin and mounted with Immumount ${ }^{\mathbb{B}}$ (Shandon, Pittsburgh, PA, USA). Tissue sections in which the specific primary antibodies were substituted with PBS or non-specific isotopic serum were used as negative controls. Caprine lymph node tissue sections were used as positive controls for all of the primary antibodies.

Immunoreactive cells were counted using 10 photomicrographs per animal at $200 \mathrm{X}$ magnification $\left(0.2 \mathrm{~mm}^{2} /\right.$ photomicrograph). Photomicrographs were used to count positively-staining cells. In liver tissue sections, areas were randomly selected, including portal spaces. In HLNs, positive cells were counted using the same methodology in both medullar and follicular areas. The results are presented as means $\pm \mathrm{SD}$. Image Proplus 4.5 biomedical software (Media Cybernetics, Silver Spring, USA) was used for cell counting. Macros were calibrated for staining intensity and cell size to include all immunostained cells.

Statistical analyses - Statistical analysis was carried out using SPSS 14.0 software for Windows (SPSS Inc, Chicago, USA). The Kolmogorov-Smirnov test was applied to decide whether distributions were parametric. The results between groups were compared using the Student $t$ test when distributions were parametric and the Mann-Whitney U test when distributions were nonparametric. Correlation studies were carried out using the Spearman correlation test for non-parametric distributions; $\mathrm{p}<0.05$ was considered significant.

\section{RESULTS}

Faecal egg counts - F. hepatica eggs began to appear in faeces nine weeks after infection. There was a good deal of individual variation in egg counts between different animals. However, egg counts rose gradually from week 9 post-infection until the end of the experi- ment, showing no statistical differences between the immunized and infected control groups.

Fluke burdens - Results for fluke burdens in group 2 and 3 are shown in Table II. The mean number of flukes recovered was $92.0 \pm 53.1$ and $101.8 \pm 61.9$ in groups 2 and 3 , respectively. No significant differences were found between groups 2 and 3. The fluke implantation rate was $46 \%$ and $50.9 \%$ in the infected control and immunized groups, respectively. It was remarkable that two goats in group 3 presented only 65 and 50 flukes, respectively, given the mean burden of this group.

Plasma levels of hepatic enzymes - Plasma levels of GLDH and GGT were within the normal range for the three groups prior to challenge (week 10). Three weeks after infection (week 13), GLDH levels were elevated in both infected groups until week 18, when a maximum level was reached (108.0 UI/L in group 2 and 108.5 UI/L in group 3), decreasing gradually until week 27 . GGT increased in week 16, reached maximum levels in week 20 (213.7 UI/L in group 2 and 116.1 UI/L in group 3) and decreased quickly to baseline levels. No significant difference was noted between groups during the study.

Histopathology and morphometric study of the liver Livers from infected animals showed superficial scars and whitish tortuous tracts, mainly involving the left hepatic lobe, whereas gross changes in the right and quadrate lobes were variable and generally less severe. Animals in groups 2 and 3 showed moderate to severe gross hepatic changes. Gall bladders and large superficial bile ducts were whitish in color and enlarged. The two animals from group 3 that died at four days post-infection (dpi) showed a few tiny whitish spots over the left hepatic lobe.

Hepatic histopathological changes consisted of fibrous perihepatitis, chronic tracts composed of an abundant infiltrate of macrophages containing brown pigment, fewer lymphocytes and plasma cells and fibrosis. In portal spaces, marked bile duct hyperplasia accompanied by fibrosis and inflammatory infiltration of eosinophils, lymphocytes and plasma cells were often found. Some animals also showed infiltration of globule

\section{TABLE II}

Results (mean \pm standard deviation) of the fluke burdens, macroscopic and microscopic liver morphometric analyses in goats from group 2 (Quillaia A) and group 3

(Schistosoma mansoni fatty acid-binding protein 14)

\begin{tabular}{lcccc}
\hline Group & $\begin{array}{c}\text { Fluke } \\
\text { burden }\end{array}$ & $\begin{array}{c}\text { Gross } \\
\text { damage } \\
\%\end{array}$ & $\begin{array}{c}\text { Microscopic }_{\text {damage }^{a}} \\
\%\end{array}$ & $\begin{array}{c}\text { Bile duct } \\
\%\end{array}$ \\
\hline 1 & - & - & $5.6 \pm 0.9$ & $0.84 \pm 0.43$ \\
2 & $92.0 \pm 53.0$ & $50.02 \pm 17.50$ & $44.48 \pm 26.82$ & $5.76 \pm 3.58$ \\
3 & $101.8 \pm 61.9$ & $21.70^{b} \pm 8.33$ & $34.46 \pm 10.18$ & $4.33 \pm 1.58$ \\
\hline
\end{tabular}

$a$ : area occupied by portal spaces in group 1 and by portal spaces, inflammatory infiltrates, fibrosis and granulomas in groups 2 and $3 ; b: \mathrm{p}<0.001$ comparing group 3 with 2 . 
leukocytes in the bile ducts. Lymphoid follicles with germinal centres were also observed in some portal spaces. Granulomas, containing an eosinophilic necrotic centre surrounded by macrophages, multinucleated giant cells, lymphocytes, plasma cells and fibrosis, were found in some animals. High individual microscopic variability was found in both infected groups. The two animals from group 3 dying at four dpi showed subcapsular granulomas containing numerous macrophages, some multinucleated giant cells, eosinophils and lymphocytes in the centre; refractile material similar to larvae cuticula could be identified (Fig. 1).

Results of gross morphometric examination of animals in groups 2 and 3 are shown in Table II. Gross morphometric observation revealed that $50 \% \pm 14.9$ (group 2) and $21.7 \% \pm 8.3$ (group 3) of the liver surface was damaged. This difference was significant $(\mathrm{p}<0.001)$. Gross liver damage showed a significant correlation $(\mathrm{p}=0.01, \mathrm{R}=-0.691)$ with experimental grouping. However, correlation between gross damage area and fluke burden was low $(R=-0.06)$ and non-significant $(p=0.79)$.

Results of microscopic liver analysis are shown in Table II. The percentage of area occupied by portal spaces and damaged areas of hepatic parenchyma (chronic tracts, cholangitis with inflammatory infiltration, fibrosis and granulomas) revealed a marked increase in the two infected groups with respect to the area of portal spaces in the uninfected control group. The percentage of damaged area in group $2(44.48 \% \pm 26.82)$ and group $3(34.46 \% \pm 10.18)$ showed no significant differences. The percentage of area occupied by bile ducts showed a very significant increase $(p<0.001)$ in the two infected groups with respect to the uninfected control group; this difference was slightly higher in group $2(5.76 \% \pm 3.58)$ compared to group $3(4.33 \% \pm 1.58)$, although the difference was not significant.

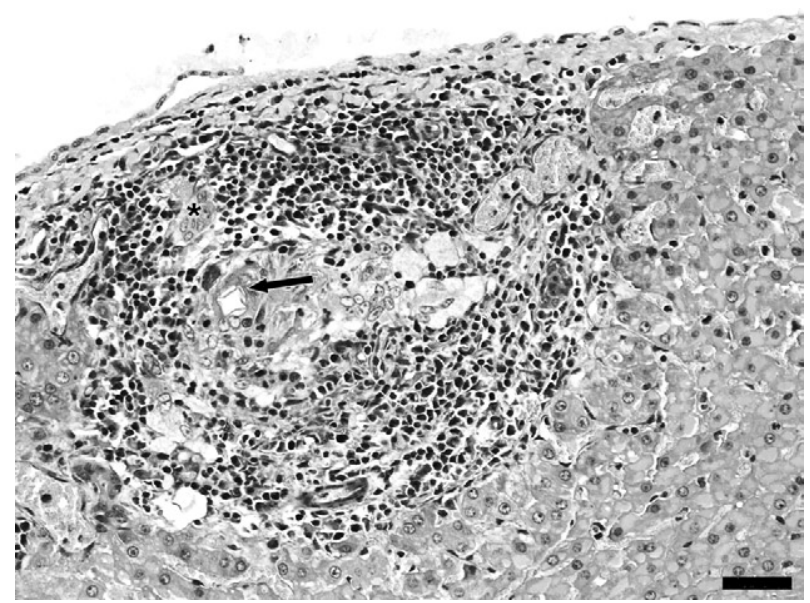

Fig. 1: liver. Subcapsular granuloma composed by numerous macrophages and lymphocytes, some multinucleate giant cells $(*)$ and eosinophils, in the center refractile material similar to larvae cuticula (arrow). Moderate subcapsular focal congestion. Haematoxylin and eosin. Bar $=50 \mu \mathrm{m}$.
Statistical correlations were found between macroscopic and microscopic liver morphology $(\mathrm{R}=0.477$, $\mathrm{p}=0.033)$ and between microscopic damage and bile duct hyperplasia $(\mathrm{R}=0.623, \mathrm{p}=0.003)$.

Histopathology and morphometric study of the HLN - The mean weight (grams) of the HLN was $1.8 \pm 0.7$, $12.57 \pm 4.68$ and $8.5 \pm 2.08$ in the negative control, infected control and immunized group, respectively. A statistically significant increase in weight was found in the two infected groups compared to the negative control group $(\mathrm{p}<0.05)$. However, no significant differences were found between infected controls and the immunized group $(\mathrm{p}=0.80)$. Grossly, HLNs from all infected goats showed severe cortex and medullar hyperplasia.

Histopathological examination of the HLN revealed marked hyperplasia of lymphoid follicles and medullary cords in infected goats. Infiltration of eosinophils was variable in paracortical areas and the medulla of infected animals, demonstrated by haemosiderin pigment in the cytoplasm of variable numbers of medullary macrophages.

The percentage of cortex occupied by lymphoid follicles was $14.85 \pm 2.79,26.36 \pm 9.36$ and $27.44 \pm 8.40$ in groups 1, 2 and 3, respectively. Statistical analysis revealed a significant increase in the area occupied by lymphoid follicles in the two infected groups with respect to uninfected controls $(\mathrm{p}<0.05)$, while no differences between the infected control and immunized group were observed $(\mathrm{p}>0.05)$.

Immunohistochemical study of the liver - Results of immunohistochemical analysis of hepatic lesions are shown in Table III. Negative controls showed occasional positive cells.

All goats from the infected groups showed severe infiltration of $\mathrm{CD} 2^{+}, \mathrm{CD}^{+}$and $\mathrm{CD}^{+}$cells, located primarily within portal spaces (Fig. 2). The ratio of $\mathrm{CD}^{+} / \mathrm{CD} 8^{+}$cells was $1.34,1.56$ and 0.98 in groups 1,2 and 3, respectively. Interestingly, there were occasional cells expressing IL- $4^{+}$ and $\mathrm{TCR}^{+}$in hepatic lesions from both infected groups. All infected animals showed a significant increase in all cell populations examined in comparison with uninfected controls $(p<0.001)$. In the rSm14-immunized group (group 3), infiltration of CD8 $8^{+}$and $\mathrm{TCR}^{+}(\mathrm{p}<0.05)$ as well as $\mathrm{CD} 2^{+}$and $\mathrm{CD} 4^{+}(\mathrm{p}<0.001)$ cells was markedly reduced in comparison with infected controls (group 2). No significant difference was noted in $\mathrm{IgG}^{+}$populations.

Immunohistochemical study of the HLN - The distribution of different cell subsets in HLN is summarized in Table III. Both infected groups showed a significant increase in all cell populations studied in comparison with uninfected controls $(p<0.001)$. In the follicular areas, the number of $\mathrm{CD} 2^{+}, \mathrm{CD} 4^{+}, \mathrm{CD} 8^{+}, \mathrm{IL}-4^{+}, \mathrm{IFN}-\gamma^{+}$and $\mathrm{TCR}^{+}$cells were markedly lower $(\mathrm{p}<0.001)$ in group 2 (infected control) than in group 3 (immunized). Similar results were observed in the medullary area for $\mathrm{CD}^{+}$ and $\mathrm{IgG}^{+}$cells $(\mathrm{p}<0.05)$ as well as for $\mathrm{CD} 2^{+}, \mathrm{CD} 8^{+}, \mathrm{IL}-4^{+}$ and IFN- $\gamma^{+}$cells $(\mathrm{p}<0.001)$. In the two infected groups, the number of IL- $4^{+}$cells was higher than the number of IFN $-\gamma^{+}$cells (Fig. 3). 
TABLE III

Mean \pm standard deviation of $T$ cell subsets per field $\left(0.2 \mathrm{~mm}^{2}\right)$ in the liver and hepatic lymph nodes of goats from group 1 (negative control), 2 (Quillaia A) and 3 (Schistosoma mansoni fatty acid-binding protein 14)

\begin{tabular}{|c|c|c|c|c|c|c|c|c|}
\hline Group & Area & $\mathrm{CD} 2$ & CD4 & CD8 & TCR & $\operatorname{IgG}$ & IFN- $\gamma$ & IL-4 \\
\hline \multirow[t]{3}{*}{1} & $\mathrm{~L}$ & $8.86 \pm 1.8$ & $6.2 \pm 1.8$ & $4.63 \pm 2.41$ & $0.3 \pm 0.1$ & $2.1 \pm 0.9$ & $0.06 \pm 0.01$ & $0.1 \pm 0.03$ \\
\hline & $\mathrm{C}$ & $38.7 \pm 8.2$ & $32.9 \pm 6.7$ & $14.7 \pm 3.1$ & $2.1 \pm 0.4$ & $2.3 \pm 0.3$ & $0.2 \pm 0.01$ & $0.02 \pm 0.01$ \\
\hline & M & $117.5 \pm 33.2$ & $69.5 \pm 11.4$ & $30.5 \pm 7.6$ & $12.4 \pm 3.3$ & $5.6 \pm 1.2$ & $0.01 \pm 0.01$ & $0.03 \pm 0.4$ \\
\hline \multirow[t]{3}{*}{2} & $\mathrm{~L}$ & $525.7 \pm 29.0$ & $246.7 \pm 55.3$ & $157.7 \pm 26.5$ & $16.9 \pm 6.6$ & $33.1 \pm 22.5$ & $0.4 \pm 0.5$ & $2.8 \pm 1.9$ \\
\hline & $\mathrm{C}$ & $1359.1 \pm 141.6$ & $699.1 \pm 124.8$ & $502.0 \pm 90.1$ & $565.3 \pm 199.8$ & $39.1 \pm 9.2$ & $5.5 \pm 3.3$ & $24.2 \pm 8.6$ \\
\hline & M & $782.7 \pm 43.2$ & $297.9 \pm 50.1$ & $370.0 \pm 41.6$ & $224.7 \pm 95.5$ & $383.4 \pm 42.6$ & $4.9 \pm 4.3$ & $19.6 \pm 2.7$ \\
\hline \multirow[t]{3}{*}{3} & $\mathrm{~L}$ & $301.2^{b} \pm 104.9$ & $100.6^{b} \pm 63.0$ & $102.4^{a} \pm 35.6$ & $5.6^{a} \pm 2.4$ & $14.2 \pm 10.9$ & $0.2^{a} \pm 0.4$ & $1.8^{b} \pm 1.8$ \\
\hline & $\mathrm{C}$ & $829.4^{b} \pm 50.5$ & $368.2^{b} \pm 47.2$ & $251.4^{b} \pm 54.6$ & $15.8^{b} \pm 12.6$ & $52.8 \pm 16.0$ & $0.60^{b} \pm 1.1$ & $2.6^{b} \pm 1.8$ \\
\hline & M & $510.0^{b} \pm 68.3$ & $198.3^{a} \pm 50.1$ & $150.6^{b} \pm 38.8$ & $14.0^{b} \pm 3.4$ & $602.9^{a} \pm 177.1$ & $0.50^{b} \pm 0.84$ & $1.0^{b} \pm 1.7$ \\
\hline
\end{tabular}

$a: \mathrm{p}<0.05$ comparing group 3 with $2 ; b: \mathrm{p}<0.01$ comparing group 3 with 2 ; C: cortical areas of hepatic lymph nodes (HLN); L: liver; M: medullar areas of HLN; statistical analyses revealed significant differences $(p<0.001)$ in group 2 and 3 compared with group 1, for all tested antibodies.

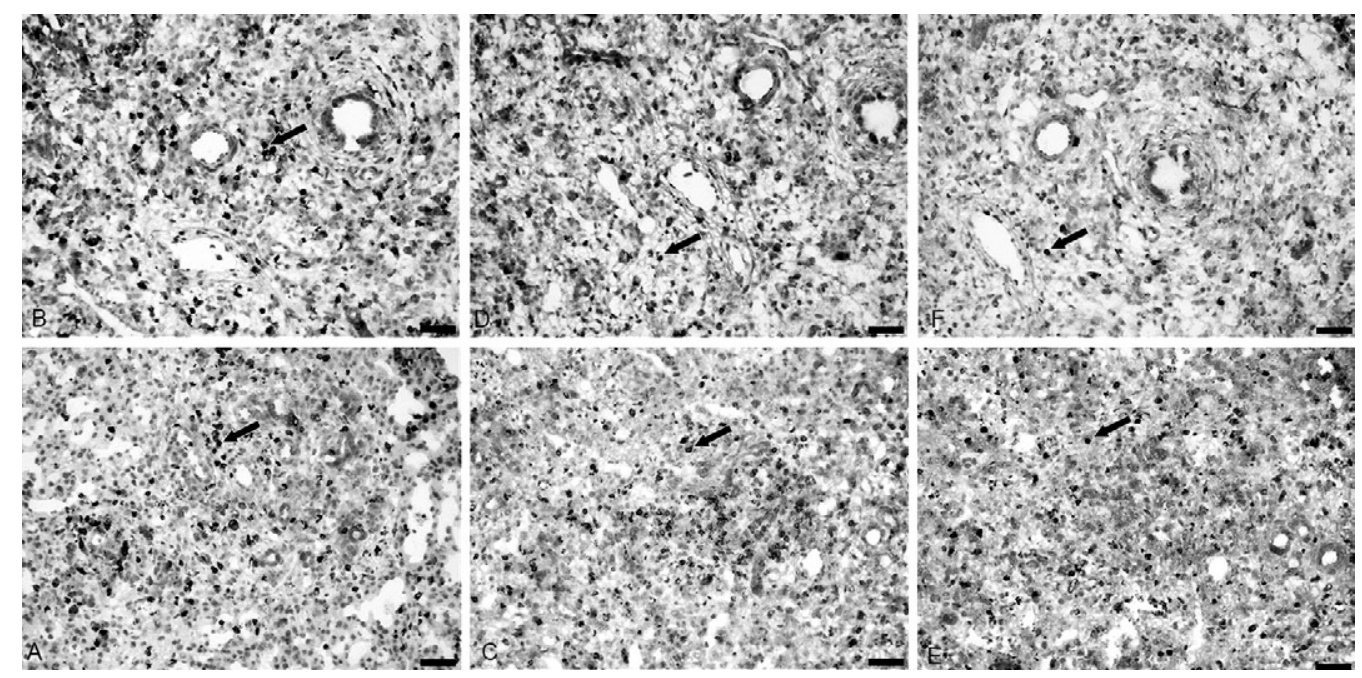

Fig. 2: liver. Portal space showing bile duct surrounded by abundant inflammatory infiltration composed mainly of CD2 $(A, B), C D 4^{+}$ $(\mathrm{C}, \mathrm{D})$ and $\mathrm{CD}^{+}(\mathrm{E}, \mathrm{F}) \mathrm{T}$ lymphocytes (arrow); group 2 (B, D, F) and group 3 (A, C, E). Avidin-biotin-peroxidase method, haematoxylin counterstain. Bar $=50 \mu \mathrm{m}$.

\section{DISCUSSION}

Goats immunized with rSm14 in Quil A did not show reduction in fluke burdens with respect to the infected control group. In a previous study using a synthetic peptide derived from the Sm14 antigen adjuvanted with Ribi and Alum, the number of flukes was $45.9 \%$ lower than the infected control, although a high degree of individual variability was recorded (Zafra et al. 2008). Additionally, Sm14 in Ribi adjuvant with Alum reduced fluke burdens by between $51.7 \%$ and $100 \%$ in mice (Tendler et al. 1996, Ramos et al. 2001, Vilar et al. 2003) as well as in sheep (Almeida et al. 2003). In all of these experiments, significant hepatic damage was also reported in vaccinated mice, sheep and goats. Therefore, the results of the present study suggest that Quil A is not a suitable adjuvant for formulating vaccines against $F$. hepatica using the Sm14 antigen. Because the best results with this antigen were obtained using Ribi, which is based on Lipid A, as an adjuvant, Sm14 is being developed as an anti-Fasciola/schistosome vaccine in formulations that include Lipid A-derived adjuvants.

In the present paper, implantation rates $(46 \%$ and $50.9 \%$ ) in the infected control and immunized group, respectively, were higher than reported in previous studies in goats (Martínez-Moreno et al. 1997, 1999, Zafra et al. 2008). However, in some experimental infections, implantation rates varying between $42-51 \%$ have been reported in sheep (Almazán et al. 2001, Ramajo et al. 2001).

Sinclair (1967) reported that after $90 \mathrm{~h}$ of infection, the first larvae reached the liver. Additionally, Presidente et al. (1974) reported that on the 7th dpi, many pale gray, 


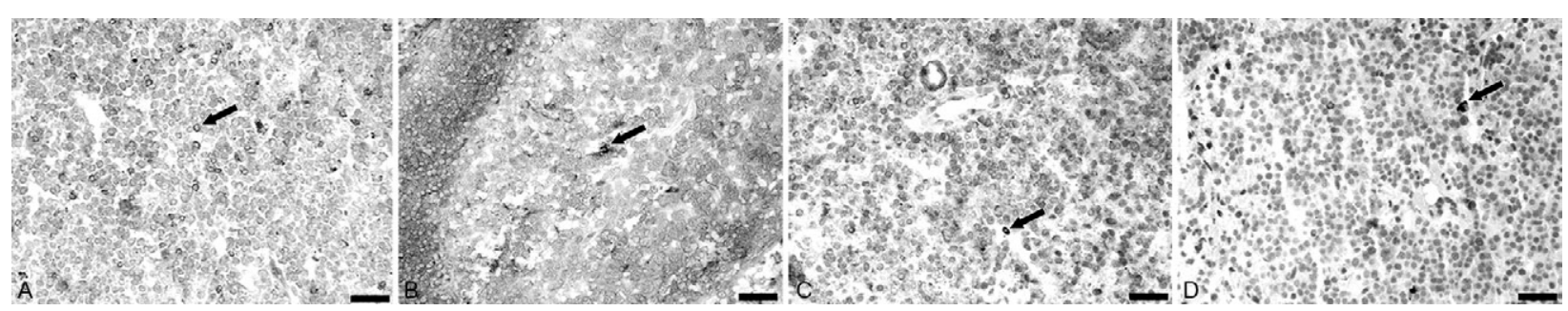

Fig. 3: medulla of a hepatic lymph node showing INF- $\gamma$ (A, B) and IL-4 (C, D) positive cells (arrow); group 2 (B, D) and group 3 (A, C). Avidinbiotin-peroxidase method, haematoxylin counterstain; $\mathrm{Bar}=50 \mu \mathrm{m}$.

irregularly shaped-areas, 4-12 $\mathrm{mm}$ in size, were found on the ventral half of the left and middle lobes. In this study, the two goats from group 3 dying at four dpi showed a few scars consistent with larval migratory lesions in the liver parenchyma. Moreover, granulomas containing cuticular debris surrounded by macrophages, eosinophils and lymphocytes indicate that the host response may have resulted in the death of some of the larvae reaching the liver parenchyma. However, results of fluke burdens indicated that only a small number of the larvae reaching the liver had died during the migratory stage, showing no significant differences with respect to the infected control group. It is of interest to investigate the local host immune response during early post-infection stages to determine the pattern of the host response in immunized and non-immunized animals due to the fact that protective response occurs during early post-infection stages in laboratory animals (Kesik et al. 2007).

Evaluation of hepatic damage in experimental fasciolosis has traditionally been assessed by visual inspection, conventional histopathology, scoring results by crosses or numbers, or even by serum levels of hepatic enzymes (Meeusen et al. 1995, Dalton et al. 1996, Martínez-Moreno et al. 1999, Mulcahy \& Dalton 2001, Raadsma et al. 2008). These methods are subjective and do not allow correlation with other factors. Recently, a morphometric study was conducted to evaluate hepatic damage in goats infected with $F$. hepatica (Zafra et al. 2008).

Gross morphometric examination of the liver showed that lesions were significantly reduced in the immunized group with respect to the infected control, despite the fact that the number of flukes in both groups was quite similar. This result could be due to a lower capacity of larvae to induce hepatic damage in the immunized goats or to the random migration of larvae. In some groups, large numbers of flukes may have randomly migrated thorough the left hepatic lobe leaving the rest of the liver virtually unaffected, while other animals with lower number of flukes presented numerous scars in both hepatic lobes. This finding may explain the lack of correlation between gross hepatic damage and fluke burdens, which was also reported in a previous trial in goats ( $\mathrm{Za}$ fra et al. 2008, Pérez-Écija et al. 2009).

In the present study, a total of $85 \mathrm{~mm}^{2}$ of liver per animal were evaluated using 10 randomly selected photomicrographs from four tissue samples, an area higher than that evaluated by Coutinho et al. (2007) and Viz- zotto et al. (2002) who used $4.9 \mathrm{~mm}^{2}$ and $1.3 \mathrm{~mm}^{2}$, respectively. The statistical correlation between the macroscopic and microscopic morphometric liver analyses and between microscopic damage and bile duct hyperplasia corroborate the idea that the sampling method used in the microscopic study was representative.

The marked enlargement of the HLN in the two infected groups with respect to uninfected controls suggests a strong immune response to $F$. hepatica infection. Histopathological and morphometric evaluation revealed that HLN hyperplasia was due mainly to hyperplasia of lymphoid follicles and medullary cords, suggesting evident robust humoral immune response, which agrees with experiments performed in sheep (Meeusen et al. 1995, Pérez et al. 2002) and goats (Martínez-Moreno et al. 1999, Zafra et al. 2009). The high infiltration of CD2 $2^{+}$, $\mathrm{CD}^{+}$and $\mathrm{CD} 8^{+}$cells into the liver and HLN in this study suggests a strong local cellular immune response against $F$. hepatica. These results agree with those previously reported in sheep (Meeusen et al. 1995, Chauvin \& Boulard 1996, Pérez et al. 2002, 2005) and goats (MartínezMoreno et al. 1999, Pérez et al. 1999, Zafra et al. 2008) chronically infected with $F$. hepatica. The increase in $\mathrm{IgG}^{+}$plasma cells in the liver and HLN of the two infected groups agrees with the increase in serum levels of $\mathrm{IgG}$ anti-rSm14 found in group 3. However, as in previous infections in sheep and goats, the host immune response in the two infected groups was non-protective as indicated by similar fluke burdens in both groups.

Reduced hepatic infiltration of $\mathrm{CD} 2^{+}, \mathrm{CD}^{+}$and $\mathrm{CD} 8^{+}$ $\mathrm{T}$ lymphocytes observed in group 3 (rSm14) compared to group 2 (Quil A) could be related to the lower hepatic damage provoked rather than the number of flukes. Similar results were recently found in goats immunized with a synthetic peptide derived from the Sm14 antigen (Zafra et al. 2009), suggesting that evaluation of the local response during early post-infection stages is important to discriminate between the host response against tissue damage caused by migrating larvae and host response against migratory larvae.

Bovine anti-IL4 and anti-IFN- $\gamma$ positively stained cells in the hepatic infiltrate and lymphocytes in HLN in the present study, confirming that both antibodies crossreacted with caprine tissues as the supplier reported. To date, expression of these antigens has only been successfully used for immunohistochemistry in fasciolosis in HLN (Zafra et al. 2009), although tissue expression 
of cytokines has been used to evaluate tissue response profiles in cattle using the antibodies used in the present study (Johnson et al. 2006). The low expression of IFN- $\gamma^{+}$and higher expression of IL4 $4^{+}$, particularly in the HLN, suggest a Th2-polarised response, a finding also reported in chronic F. hepatica infections in goats (Zafra et al. 2009), cattle (Waldvogel et al. 2004) and sheep (Zhang et al. 2005).

Despite the similarity of fluke burdens, we observed a significant reduction in both gross hepatic damage and local inflammatory infiltrates in animals immunized with Sm14 in Quil A. We speculate that a reduced immune response induced in immunized animals was responsible for this action. This effect could be due to inhibited function of Sm14 in the parasite, compromising the uptake, transport and compartmentalization of host-derived fatty acids due to the organized immune response against this protein. This response was not able to kill the parasite per se, however it produced a desirable result in an immunization-based control program, which could be improved with further studies.

In conclusion, goats immunized with $\mathrm{rSm} 14$ in Quil A and subsequently infected with $F$. hepatica did not show any reduction in fluke burdens and faecal egg counts, but gross hepatic lesions were significantly reduced in the immunized group with respect to the infected control group. A strong local immune response, represented by infiltration of Th2-polarised $\mathrm{CD} 2^{+}, \mathrm{CD}^{+}$and $\mathrm{CD} 8^{+}$ T lymphocytes, was recorded in the liver and HLN of both infected groups and was more severe in the infected control group than in the immunized group. However, in both groups, the host response was non-protective. Further studies are needed to investigate the nature of the local immune response in the context of new vaccine formulations in a Lipid A-based adjuvant to evaluate the potential usefulness of Sm14 antigen as a vaccine for fasciolosis in ruminants.

\section{REFERENCES}

Almazán C, Avila G, Quiroz H, Ibarra F, Ochoa P 2001. Effect of parasite burden on the detection of Fasciola hepatica antigens in sera and feces of experimentally infected sheep. Vet Parasitol 97: 101-112.

Almeida MS, Torloni H, Lee-Ho P, Vilar MM, Thaumaturgo N, Simpson AJ, Tendler M 2003. Vaccination against Fasciola hepatica infection using a Schistosoma mansoni defined recombinant antigen, Sm14. Parasite Immunol 25: 135-137.

Chauvin A, Boulard C 1996. Local immune response to experimental Fasciola hepatica infection in sheep. Parasite 3: 209-215.

Coutinho EM, Silva FL, Barros AF, Araújo RE, Oliveira SA, Luna CF, Barbosa AA Jr, Andrade ZA 2007. Repeated infections with Schistosoma mansoni and liver fibrosis in undernourished mice. Acta Trop 101: 15-24.

Dalton JP, McGonigle S, Rolph TP, Andrews SJ 1996. Induction of protective immunity in cattle against infection with Fasciola hepatica by vaccination with cathepsin L proteinases and with hemoglobin. Infect Immun 64: 5066-5074.

Dalton JP, Neill SO, Stack C, Collins P, Walshe A, Sekiya M, Doyle S, Mulcahy G, Hoyle D, Khaznadji E, Moiré N, Brennan G, Mousley A, Kreshchenko N, Maule AG, Donnelly SM 2003. Fasciola hepatica cathepsin L-like proteases: biology, function, and potential in the development of first generation liver fluke vaccines. Int J Parasitol 33: 1173-1181.
Johnson L, Gough J, Spencer Y, Hewinson G, Vordermeier M, Wangoo A 2006. Immunohistochemical markers augment evaluation of vaccine efficacy and disease severity in bacillus CalmetteGuerin (BCG) vaccinated cattle challenged with Mycobacterium bovis. Vet Immunol Immunopathol 111: 219-229.

Kesik M, Jedlina-Panasiuk L, Kozak-Cieszczyk M, Płucienniczak A, Wedrychowicz H 2007. Enteral vaccination of rats against Fasciola hepatica using recombinant cysteine proteinase (cathepsin L1). Vaccine 25: 3619-3628.

Martínez-Moreno A, Jiménez-Luque V, Moreno T, Redondo ES, de las Mulas JM, Pérez J 1999. Liver pathology and immune response in experimental Fasciola hepatica infections of goats. Vet Parasitol 82: 19-33.

Martínez-Moreno A, Martínez-Moreno FJ, Acosta I, Gutiérrez PN, Becerra C, Hernández S 1997. Humoral and cellular immune responses to experimental Fasciola hepatica infections in goats. Parasitol Res 83: 680-686.

Mas-Coma S, Bargues MD, Valero MA 2005. Fascioliasis and other plant-borne trematode zoonoses. Int J Parasitol 35: 1255-1278.

Meeusen E, Lee CS, Rickard MD, Brandon MR 1995. Cellular responses during liver fluke infection in sheep and its evasion by the parasite. Parasite Immunol 17: 37-45.

Meeusen EN, Piedrafita D 2003. Exploiting natural immunity to helminth parasites for the development of veterinary vaccines. Int $J$ Parasitol 33: 1285-1290.

Mendes RE, Pérez-Ecija RA, Zafra R, Buffoni L, Martínez-Moreno A, Dalton JP, Mulcahy G, Pérez J 2010. Evaluation of hepatic changes and local and systemic immune responses in goats immunized with recombinant Peroxiredoxin (Prx) and challenged with Fasciola hepatica. Vaccine 28: 2832-2840.

Moll L, Gaasenbeek CP, Vellema P, Borgsteede FH 2000. Resistance of Fasciola hepatica against triclabendazole in cattle and sheep in The netherlands. Vet Parasitol 91: 153-158.

Moser D, Tendler M, Griffiths G, Klinkert MQ 1991. A 14-kDa Schistosoma mansoni polypeptide is homologous to a gene family of fatty acid binding proteins. $J$ Biol Chem 266: 8447-8454.

Mulcahy G, Dalton JP 2001. Cathepsin L proteinases as vaccines against infection with Fasciola hepatica (liver fluke) in ruminants. Res Vet Sci 70: 83-86.

Navarro JA, Caro MR, Seva J, Rosillo MC, Gomez MA, Gallego MC 1996. Study of lymphocyte subpopulations in peripheral blood and secondary lymphoid organs in the goat using monoclonal antibodies to surface markers of bovine lymphocytes. Vet Immunol Immunopathol 51: 147-156.

Overend DJ, Bowen FL 1995. Resistance of Fasciola hepatica to triclabendazole. Aust Vet J 72: 275-276.

Pérez J, de las Mulas JM, De Lara FC, Gutierrez-Palomino PN, Becerra-Martel C, Martínez-Moreno A 1998. Immunohistochemical study of the local immune response to Fasciola hepatica in primarily and secondarily infected goats. Vet Immunol Immunopathol 64: 337-348.

Pérez J, Martín de las Mulas J, Carrasco L, Gutierrez PN, MartínezCruz MS, Martínez-Moreno A 1999. Pathological and immunohistochemical study of the liver and hepatic lymph nodes in goats infected with one or more doses of Fasciola hepatica. J Comp Pathol 120: 199-210.

Pérez J, Ortega J, Bravo A, Díez-Baños P, Morrondo P, Moreno T, Martínez-Moreno A 2005. Phenotype of hepatic infiltrates and hepatic lymph nodes of lambs primarily and challenge infected with Fasciola hepatica, with and without triclabendazole treatment. Vet Res 36: 1-12. 
Pérez J, Ortega J, Moreno T, Morrondo P, López-Sández C, MartínezMoreno A 2002. Pathological and immunohistochemical study of the liver and hepatic lymph nodes of sheep chronically reinfected with Fasciola hepatica, with or without triclabendazole treatment. J Comp Pathol 127: 30-36.

Pérez-Écija RA, Mendes RE, Zafra R, Buffonni L, Martínez-Moreno A, Pérez J 2009. Pathological and parasitological protection in goats immunised with recombinant cathepsin L1 and challenged with Fasciola hepatica. Vet $J$ [Epub ahead of print].

Presidente PJ, McCraw BM, Lumsden JH 1974. Early pathological changes associated with Fasciola hepatica infections in whitetailed deer. Can J Comp Med 38: 271-279.

Raadsma HW, Kingsford NM, Suharyanta, Spithill TW, Piedrafita D 2008. Host responses during experimental infection with Fasciola gigantica and Fasciola hepatica in Merino sheep II. Development of a predictive index for Fasciola gigantica worm burden. Vet Parasitol 154: 250-261.

Ramajo V, Oleaga A, Casanueva P, Hillyer GV, Muro A 2001. Vaccination of sheep against Fasciola hepatica with homologous fatty acid binding proteins. Vet Parasitol 97: 35-46.

Ramos CRR, Vilar MM, Nascimento ALTO, Ho PL, Thaumaturgo N, Edelenyi R, Almeida M, Dias WO, Diogo CM, Tendler M 2001. r-Sm14 - pRSETA efficacy in experimental animals. Mem Inst Oswaldo Cruz 96 (Suppl.): 131-135.

Sinclair KB 1967. Pathogenesis of Fasciola and the other liver-flukes. Helminthol Abstracts 36: 115-134.

Spithill TW, Smooker PM, Sexton JL, Bozas E, Morrison CA, Creaney J, Parsons JC 1999. Development of vaccines against Fasciola hepatica. In JP Dalton (ed.), Fasciolosis, CABI Publising, Cambridge, p. 465-526.

Tendler M, Brito CA, Vilar MM, Serra-Freire N, Diogo CM, Almeida MS, Delbem AC, Da Silva JF, Savino W, Garratt RC, Katz N,
Simpson AS 1996. Schistosoma mansoni fatty acid-binding protein, $\mathrm{Sm} 14$, is the potential basis of a dual-purpose anti-helminth vaccine. Proc Natl Acad Sci USA 93: 269-273.

Tendler M, Simpson AJ 2008. The biotechnology-value chain: development of Sm14 as a schistosomiasis vaccine. Acta Trop 108: 263-266.

Tendler M, Vilar MM, Brito CA, Freire NM, Katz N, Simpson A 1995. Vaccination against schistosomiasis and fascioliasis with the new recombinant antigen Sm14: potential basis of a multi-valent antihelminth vaccine? Mem Inst Oswaldo Cruz 90: 255-256.

Vilar MM, Barrientos F, Almeida M, Thaumaturgo N, Simpson A, Garratt R, Tendler M 2003. An experimental bivalent peptide vaccine against schistosomiasis and fascioliasis. Vaccine 22: 137-144.

Vizzotto L, Vertemati M, Gambacorta M, Sabatella G, Spina V, Minola E 2002. Analysis of histological and immunohistochemical patterns of the liver in posthepatitic and alcoholic cirrhosis by computerized morphometry. Mod Pathol 15: 798-806.

Waldvogel AS, Lepage MF, Zakher A, Reichel MP, Eicher R, Heussler VT 2004. Expression of interleukin 4, interleukin 4 splice variants and interferon gamma mRNA in calves experimentally infected with Fasciola hepatica. Vet Immunol Immunopathol 97: 53-63.

Zafra R, Buffoni L, Martínez-Moreno A, Pérez-Ecija A, MartinezMoreno FJ, Pérez J 2008. A study of the liver of goats immunized with a synthetic peptide of the Sm14 antigen and challenged with Fasciola hepatica. J Comp Pathol 139: 169-176.

Zafra R, Buffoni L, Pérez-Ecija RA, Mendes RE, Martínez-Moreno A, Martínez-Moreno FJ, Pérez J 2009. Study of the local immune response to Fasciola hepatica in the liver and hepatic lymph nodes of goats immunised with a peptide of the Sm14 antigen. Res Vet Sci 87: 226-232.

Zhang WY, Moreau E, Hope JC, Howard CJ, Huang WY, Chauvin A 2005. Fasciola hepatica and Fasciola gigantica: comparison of cellular response to experimental infection in sheep. Exp Parasitol 111: 154-159. 